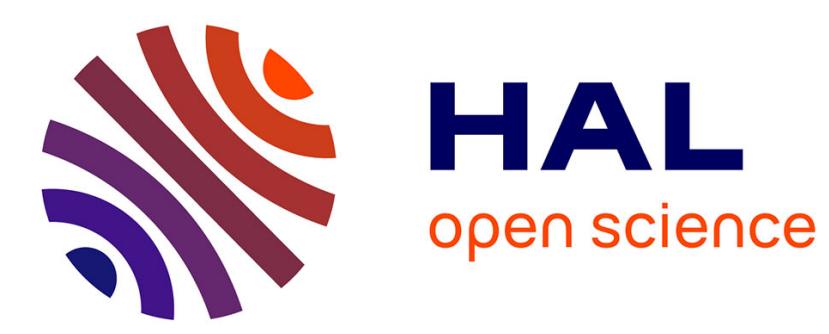

\title{
Numerical Investigation on the Spectral Broadening of Acoustic Waves by a Turbulent Layer \\ Vincent Clair, Gwenael Gabard
}

\section{To cite this version:}

Vincent Clair, Gwenael Gabard. Numerical Investigation on the Spectral Broadening of Acoustic Waves by a Turbulent Layer. 22nd AIAA/CEAS Aeroacoustics Conference, 2016, Lyon, France. 10.2514/6.2016-2701 . hal-02085971

\section{HAL Id: hal-02085971 https://hal.science/hal-02085971}

Submitted on 16 Apr 2019

HAL is a multi-disciplinary open access archive for the deposit and dissemination of scientific research documents, whether they are published or not. The documents may come from teaching and research institutions in France or abroad, or from public or private research centers.
L'archive ouverte pluridisciplinaire HAL, est destinée au dépôt et à la diffusion de documents scientifiques de niveau recherche, publiés ou non, émanant des établissements d'enseignement et de recherche français ou étrangers, des laboratoires publics ou privés. 


\title{
Numerical Investigation on the Spectral Broadening of Acoustic Waves by a Turbulent Layer
}

\author{
V. Clair* and G. Gabard ${ }^{\dagger}$ \\ Institute of Sound and Vibration Research, University of Southampton, UK.
}

\begin{abstract}
When acoustic waves are propagating through turbulence, a scattering phenomenon occurs leading to a broadening of the acoustic spectrum. This phenomenon has been observed experimentally for a harmonic source radiating through the shear layer of a cold jet at low speed. The observed spectra are displaying a characteristic shape consisting in a more or less reduced peak amplitude at the source frequency, surrounded by sidebands (also called haystacks). The levels and width of these sidebands appear to be evolving with the source and flow parameters. This spectral broadening is studied in this paper for a simplified configuration consisting in a monopole radiation propagating through a turbulent layer with a constant thickness and convected by a uniform mean flow. The numerical method used relies on a finite difference code solving the linearized Euler equations in the time domain. The turbulence is synthesized using a stochastic method based on the filtering of white noise to impose prescribed statistical properties to the turbulent velocity field. These turbulent fluctuations are then added to the steady mean flow to form an unsteady base flow around which the Euler equations are linearized. This new set of equations contains terms involving products between the turbulent and acoustic fluctuations, which are responsible for the scattering. In this paper, the trends deduced from this numerical study can be compared to analytical models and experimental data obtained for a jet shear layer. They can also be related to the trends observed in a previous study on the scattering of sound by a single convected vortex.
\end{abstract}

\section{Introduction}

A scattering phenomenon occurs when acoustic waves propagate through a volume of turbulence, leading to a spatial and spectral redistribution of the acoustic energy. The first studies of this phenomenon have been realized by Lighthill ${ }^{25}$ and Kraichnan ${ }^{23}$ using Lighthill's acoustic analogy and assuming that the scattering is weak (meaning that only a small proportion of the acoustic energy is scattered). The work of Lighthill was focused on the spatial scattering of the acoustic energy, while Kraichnan also expressed the frequency spectrum, particularly for isotropic turbulence. Kinetic equations have been derived by Howe, ${ }^{22}$ in which the different terms can be linked either to the spatial or spectral scattering. These equations are able to consider multiple scattering whereas the previous models relied on the hypothesis of single scattering across the turbulent volume.

Acoustic scattering by turbulence also received attention in the context of atmospheric propagation. Brown $^{2}$ and Brown \& Clifford ${ }^{3,4}$ developed a model based on an inhomogeneous Helmholtz equation with a source term involving the turbulent velocity and temperature fluctuations. Ostashev et al. ${ }^{27}$ and Salomons et al. ${ }^{29}$ studied the sound propagation near an impedance ground in an atmosphere with temperature and velocity fluctuations using a model based on the parabolic equation. Dallois et al. ${ }^{11}$ solved a vector parabolic equation numerically to consider the propagation through an inhomogeneous turbulent atmosphere. Goedecke et al. ${ }^{20}$ proposed a semi-analytical model based on the scattering by a single eddy. A turbulent volume is split into cells of different sizes containing an eddy, and the scattering by the whole volume is obtained by computing the scattering by each eddy. A similar approach has been proposed by Wilson et

\footnotetext{
${ }^{*}$ Research Fellow, Institute of Sound and Vibration Research, University of Southampton. SO17 1BJ Southampton, UK. V.J.Clair@soton.ac.uk

†Associate Professor, Institute of Sound and Vibration Research, University of Southampton. SO17 1BJ Southampton, UK. AIAA Member.
} 
al., ${ }^{32}$ using a quasi-wavelet method to represent the turbulent velocity field over a barrier and to study the scattering of waves behind the barrier. More recently, Cheinet et al. ${ }^{9}$ developed a method using finite difference in the time domain to solve a two equations system and investigate the scattering by an atmospheric turbulence composed of temperature fluctuations only. The fluctuations are synthesized stochastically and the results are averaged over a set of realizations. A similar method has been developed by Ehrhardt et al. ${ }^{13}$ to study the scattering of a plane wave through strong turbulent velocity fluctuations, solving the linearized Euler equations.

The propagation of acoustic waves through the shear layer of an open wind tunnel or an aero-engine jet is another area of interest for the study of acoustic scattering. These configurations have been studied experimentally by Candel et al. ${ }^{7,8}$ and more recently in the framework of the project GARTEUR AD/AG-

$50,{ }^{24,30}$ considering a harmonic source on the jet centerline. The results highlighted a specific shape of the acoustic spectra consisting in an attenuated peak at the source frequency surrounded by sidebands (also called haystacks). Analytical models have also been developed for such configurations, notably by Campos, ${ }^{5,6}$ who concluded that widening the shear layer could significantly reduce tonal components propagating through the exhaust of a jet engine. Guédel ${ }^{21}$ proposed an analysis of spectral broadening following the approach of Lighthill ${ }^{25}$ under the Born approximation. The results include an estimation of the ratio of scattered to total energy received by an observer, as well as an investigation on the amplitude and phase fluctuations of the scattered field. More recently, Powles et al. ${ }^{28}$ and McAlpine et al. ${ }^{26}$ developed a model to predict the haystacking of turbine tones by either frozen or evolving turbulence, considering realistic shear layer velocity profiles and turbulence characteristics. Variations of the source and flow parameters showed behaviors of the acoustic spectra that are consistent with experimental results. The first attempts of numerical calculation of the spectral broadening by a jet shear layer have been performed by Ewert et al. ${ }^{16,17}$ and showed promising results in the ability of the method to recover some features of the spectral broadening. The numerical method relied on the high order finite difference solver PIANO and the stochastic method RPM to synthesize the turbulent velocity fluctuations of the shear layer. Finally, a first large eddy simulation study was performed by Bennaceur et al. ${ }^{1}$ on the scattering of acoustic waves from a harmonic point source by a turbulent mixing layer. Several source frequencies were considered and the comparison with existing experimental results presenting similar scattering parameters showed good predictions of the positions of the haystacks and of the decay of the spectra.

The work that is presented in this paper uses the same method as Ewert et al. ${ }^{16,17}$ to study the scattering of a monopole radiation by an idealized turbulent layer with constant width and convected by a uniform mean flow. This configuration discards the refraction effects by the mean flow and the spreading of the turbulent layer observed for a jet shear layer (leading to an evolution of the scattering along the axis). Moreover, this configuration can be considered as the next step (in terms of complexity) to the recent work ${ }^{10}$ on the scattering by a single vortex convected by a uniform mean flow. In the present paper, results are presented for several source frequencies and Mach numbers of the mean flow. The shapes of the spectra display similarities with previous studies of the spectral broadening by a shear layer, and also strong similarities with the scattering by a single vortex for low frequency sources. The evolution of these spectra with the parameters considered are in agreement with previous experimental studies apart from some differences linked to the spectral content of the turbulence or to the spreading of the shear layer, which is not accounted here.

\section{Numerical method}

The PIANO solver, developed by the DLR, is used in order to solve the linearized Euler equations ( $L E E)$ in the time domain. This solver has been used in order to study a vast range of problems in acoustics, notably including interactions with turbulence via a coupling with a stochastic turbulence generation method ( $R P M$ or $f R P M) .{ }^{14,15,18}$ In this paper the $R P M$ is used in order to generate the unsteady velocity fluctuations of the turbulent layer. These fluctuations are generated using an auxiliary grid where random particles are introduced, convected and filtered in space (and eventually time) with a filter defined to enforce prescribed correlations of the velocity. Here the filter ensures Gaussian spatial correlations and no time decorrelation (frozen turbulence). More details on the $R P M$ can be found in [14-18].

In order to capture the interaction between the incident acoustic field and the convected vortex, the equations have to contain terms involving products between the acoustic fluctuations and the turbulent flow. A set of equations has been developed by Ewert et al. ${ }^{17}$ by considering small perturbations $\left(\rho^{\prime}, \boldsymbol{u}^{\prime}, p^{\prime}\right)$ over 
an unsteady base flow $(\rho, \boldsymbol{u}, p)$ instead of the usual steady mean flow $\left(\rho_{0}, \boldsymbol{u}_{0}, p_{0}\right)$ used in the LEE. This unsteady base flow is then split into a steady mean part $\Psi_{0}$ and a fluctuating part $\Psi_{t}$ (here the turbulent velocities): $\Psi(\boldsymbol{x}, t)=\Psi_{0}(\boldsymbol{x})+\Psi_{t}(\boldsymbol{x}, t)$, where $\Psi$ is $\rho, \boldsymbol{u}$ or $p$.

The equations written using this decomposition are similar to the LEE:

$$
\begin{aligned}
& \frac{\partial \rho^{\prime}}{\partial t}+\boldsymbol{u}_{0} . \nabla \rho^{\prime}+\boldsymbol{u}^{\prime} \cdot \nabla \rho_{0}+\rho_{0} \nabla \cdot \boldsymbol{u}^{\prime}+\rho^{\prime} \nabla \cdot \boldsymbol{u}_{0}=h_{1} \\
& \frac{\partial \boldsymbol{u}^{\prime}}{\partial t}+\left(\boldsymbol{u}_{0} . \nabla\right) \boldsymbol{u}^{\prime}+\left(\boldsymbol{u}^{\prime} \cdot \nabla\right) \boldsymbol{u}_{0}+\frac{\nabla p^{\prime}}{\rho_{0}}-\frac{\nabla p_{0} \rho^{\prime}}{\rho_{0}^{2}}=\boldsymbol{h}_{2} \\
& \frac{\partial p^{\prime}}{\partial t}+\boldsymbol{u}_{0} . \nabla p^{\prime}+\boldsymbol{u}^{\prime} \cdot \nabla p_{0}+\gamma p_{0} \nabla \cdot \boldsymbol{u}^{\prime}+\gamma p^{\prime} \nabla \cdot \boldsymbol{u}_{0}=h_{3}
\end{aligned}
$$

where all the interaction terms between the acoustic field and the unsteady flow have been moved to the right hand side in $\left(h_{1}, \boldsymbol{h}_{\mathbf{2}}, h_{3}\right)$. Some assumptions can be made in order to simplify these right hand side terms. First, the unsteady part of the mean flow is assumed to be divergence-free $\left(\nabla \cdot \boldsymbol{u}_{t}=0\right)$, which means that it does not introduce additional sound sources. This hypothesis is ensured when velocity fluctuations are generated with the RPM. The second assumption introduced by Ewert et al. ${ }^{17}$ is that the scattering of the incident acoustic field is mainly induced by the unsteady velocity fluctuations. Thus the effects of the unsteady density and pressure fluctuations are negligible. This assumption has been assessed numerically in [10] for a case of scattering of a plane wave by a steady vortex. Finally, with these simplifications, the right hand side terms of Eq. (1) writes:

$$
\begin{aligned}
& h_{1}=-\boldsymbol{u}_{t} \cdot \nabla \rho^{\prime} \\
& h_{2}=-\left(\boldsymbol{u}_{t} \cdot \nabla\right) \boldsymbol{u}^{\prime}-\left(\boldsymbol{u}^{\prime} \cdot \nabla\right) \boldsymbol{u}_{t} \\
& h_{3}=-\boldsymbol{u}_{t} \cdot \nabla p^{\prime}
\end{aligned}
$$

Ewert et al. ${ }^{16,17}$ suffered from growing numerical instabilities when trying to solve these equations, because of the terms of Eq. (1) involving mean flow gradients. In the present paper, since the steady mean flow considered is uniform, therefore there is no gradients and the equations do not require any specific treatment.

The spatial discretization of this set of equations is realized in PIANO using the 7 points stencil, $4^{\text {th }}$ order DRP finite differences scheme by Tam \& Webb, ${ }^{31}$ and an explicit $8^{\text {th }}$ order filter is applied in order to prevent high frequency spurious oscillations to appear in the computational domain. The time integration is performed using a $4^{\text {th }}$ order Runge-Kutta time marching scheme with 4 stages. A coordinate transformation is applied in order to deal with curvilinear meshes encountered for complex geometries, and the solver is parallelized using the MPI library. In the computations realized in this paper, the acoustic sources considered are monopoles located relatively far from the vortex path. In order to reduce the computational effort, the monopole radiation is introduced into the numerical domain through a sponge layer located next to a boundary (the lower boundary in this work).

\section{Configuration studied}

The turbulent layer considered has a constant width defined by the length scale $\delta$, is centered on the $y$-axis and has a length $20 \delta(-10 \delta \leq x \leq 10 \delta)$. The $R P M$ is used to generate frozen turbulence with Gaussian spatial correlations and a constant integral length scale $\Lambda=\delta / 4$. The turbulent kinetic energy is maximum at $y=0$ with a turbulent intensity $T_{I}=15 \%$, and it decreases following a Gaussian progression with $k_{t} \approx k_{t, \max } / 4$ at $y= \pm \delta / 2$. The mean flow is uniform, oriented in the $+x$-direction and the the Mach numbers considered are $\mathrm{M}=0.088,0.176,0.352$. The acoustic source is a harmonic monopole located at $x=0$ and $y=-7.5 \delta$. The source frequencies $f_{0}=c_{0} / \lambda_{0}$ considered in this paper are comprised in the range $c_{0} / 2 \delta \leq f_{0} \leq 8 c_{0} / \delta$.

A schematic of the numerical domain used for this first calculation is presented in Fig. 1. The grey area represents the auxiliary grid used by the $R P M$ to generate the turbulence. The cell spacing on this auxiliary mesh is uniform and ensures $\Lambda \geq 6 \Delta x_{R P M}$ as recommended by Dieste \& Gabard. ${ }^{12}$ The Gaussian filter applied to each random particle in the $R P M$ has been shown to receive contributions from the other particles located at distances $r \leq 2 \Lambda$. Thus, to reproduce the correlations correctly over the width $\delta$, the auxiliary 
grid extends up to $y= \pm(\delta / 2+2 \Lambda)$. Random particles are initialized at every nodes of the auxiliary grid and new particles are introduced every $\Delta t_{R P M}=\Delta x_{R P M} / U_{0}$.

The computational domains used for the PIANO calculations are uniformly meshed for $-10 \delta \leq x \leq 10 \delta$ and $-3 \delta \leq y \leq 8 \delta$ with a cell size ensuring a correct discretization of the source acoustic wavelengths in every directions. Thus, for the low frequency cases (up to $f_{0}=2 c_{0} / \delta$ ), the cell size of the CAA mesh is the same as the cell size of the RPM auxiliary grid. Additional sponge layers are defined to dissipate fluctuations before they reach the boundaries. At the lower boundary, the sponge layer is composed of 50 uniform cells and is also used to introduce the monopole radiation in the domain. The sponge layers defined at the upstream and upper boundaries also comprise 50 cells with no stretching for the low frequency cases, and a slight stretching is used for the high frequency cases to increase the size of the sponge layer. The sponge layer at the downstream boundary is defined differently depending on the frequency of the source considered since an instability is developing within the turbulent layer when acoustic waves are propagating through it. This instability is convected by the mean flow and can generate spurious low frequency pressure waves if it impacts the downstream boundary. For low frequency sources, these spurious waves can contaminate the spectra in the frequency range of interest. Thus, for source frequencies up to $f_{0}=2 c_{0} / \delta$, the downstream sponge layer is composed of 150 uniform cells. For higher frequencies, in order to limit the computational cost of the RPM interpolations, the downstream sponge layer is defined by 50 stretched cells. Additionally, Tam's radiation and outflow boundary conditions ${ }^{31}$ are used with a radiation center defined at the position of the monopole source. A circular array of microphones with a radius $r=15 \delta$, centered on the source position is defined for angles between $50^{\circ} \leq \theta \leq 130^{\circ}$ (with $\theta=0^{\circ}$ oriented in the $+x$-direction). The time-step of the computations is chosen to ensure a value of the CFL criterion less than 1 , and the computations are run over at least $10^{6}$ iterations to obtain converged statistical properties of the turbulence.

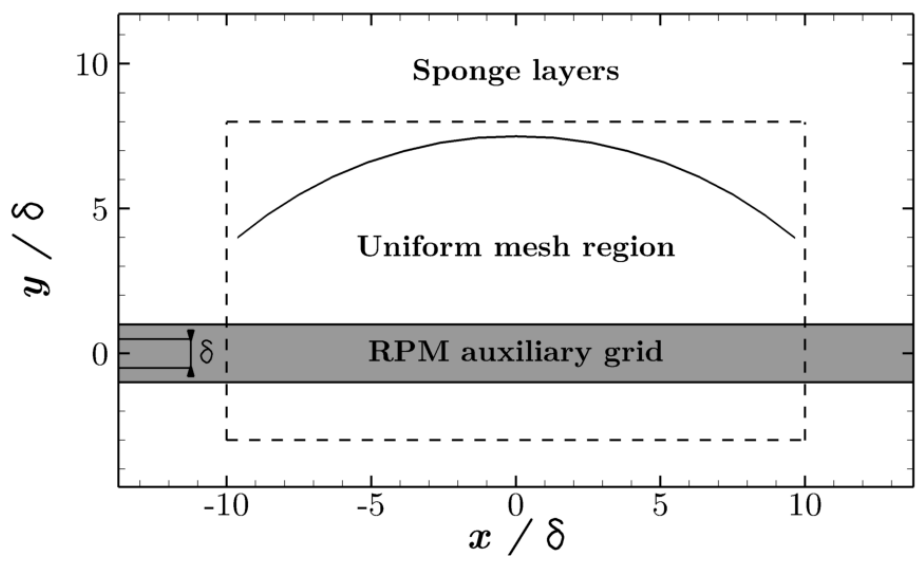

Figure 1: Schematic of the computational domain.

An example of a turbulent velocity field generated with the $R P M$, using the meshing and time discretization parameters defined above for low frequency cases $\left(\Delta x_{C A A}=\Delta x_{R P M}\right)$ is presented in Fig. 2(a). The corresponding spatial correlations $R_{x x}\left(r_{x}\right)$ and $R_{y y}\left(r_{x}\right)$ as well as the one-dimensional spectra $E_{x x}\left(\kappa_{x}\right)$ and $E_{y y}\left(\kappa_{x}\right)$ (where $E_{i j}\left(\kappa_{x}\right)$ is defined as the Fourier transform of $2 R_{i j}\left(r_{x}\right)$ ) are presented in Fig. 2(b) and show a good reproduction of the the analytical solution.

The distribution of turbulent kinetic energy through the turbulent layer is presented in Fig. 3 for two computations with different source frequencies and thus different CAA cell sizes. If the imposed distribution was defined as strictly Gaussian, the RPM would tend to underestimate the maximum turbulent kinetic energy in the center of the turbulent layer. In order to compensate for that, the imposed distribution is defined with a plateau at $k_{t, \max }$ around the centerline of the layer as shown in Fig. 3. Note that, due to a slight mistake in the definition of the imposed turbulent kinetic energy distribution, the value of $k_{t}$ at $y= \pm \delta / 2$ is closer to $0.3 k_{t, \max }$ than to the value of $k_{t, \max } / 4$ mentioned above. For the high frequency simulations (when $\Delta x_{C A A}<\Delta x_{R P M}$ ), it seems that the interpolations between the CAA and the $R P M$ grids are affecting the turbulence and, as a result, the maximum turbulent kinetic energy is underestimated (the spectra are also slightly affected at high frequency). 


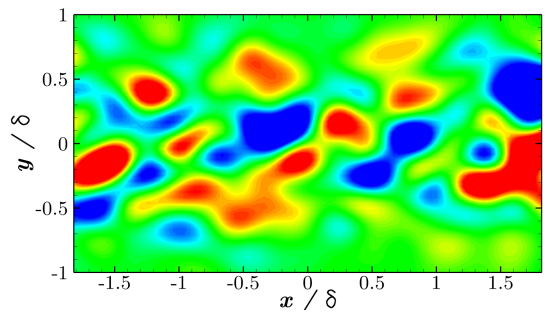

(a)
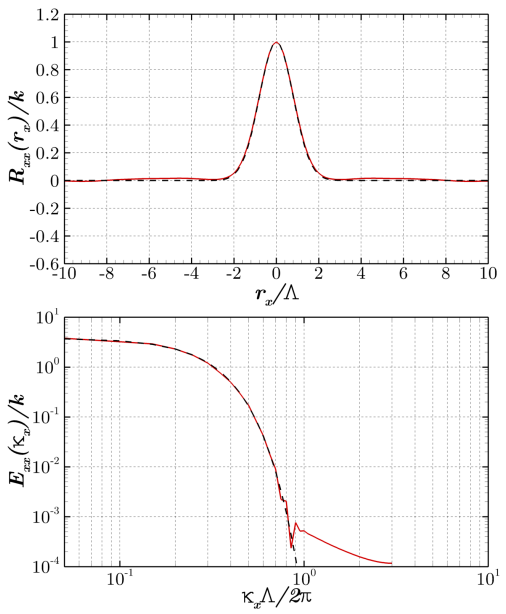

(b)
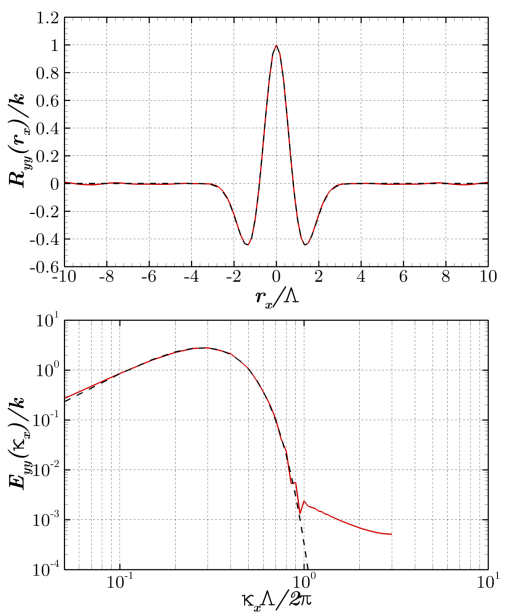

Figure 2: Example of a turbulent field synthesized using the $R P M$. (a) Snapshot of the $u_{t} / c_{0}$ velocity fluctuation (levels between \pm 0.025$)$. (b) Correlations and one-dimensional spectra: $(-) R P M$ and (- - -) analytical solutions.

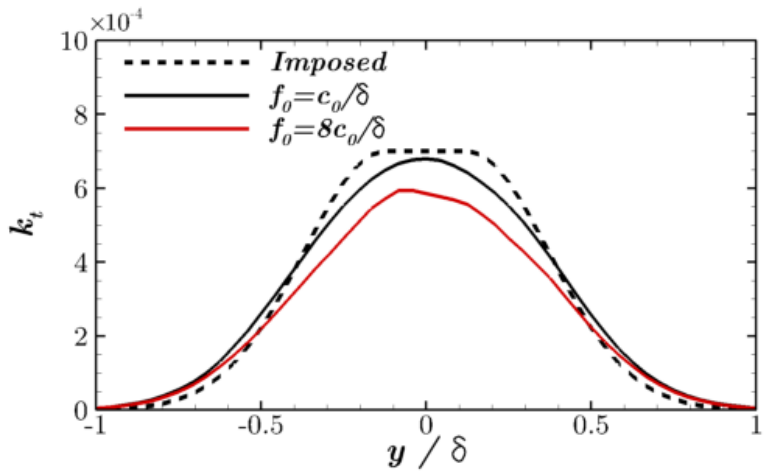

Figure 3: Turbulent kinetic energy through the turbulent layer synthesized using the RPM for two computational cases with different CAA cell sizes.

\section{Acoustic results}

\section{IV.A. Effects of the source frequency on the scattering}

The results of the simulations performed for a range of frequencies from $f_{0}=c_{0} / 2 \delta$ to $f_{0}=8 c_{0} / \delta$ with a convection mean flow at $\mathrm{M}=0.176$ are presented in this section. Fig. 4 presents snapshots of the total and scattered pressure fluctuations in the computational domain, as well as contours of the turbulent velocity, for a low frequency case $\left(f_{0}=c_{0} / \delta\right)$. The alteration of the monopole wavefronts is slightly visible on the total pressure field. The scattered pressure field is obtained by running a similar computation without the turbulent layer, and by subtracting the pressure field without turbulence from the field with turbulence. The contour values for the pressure fluctuations in Fig. 4 are normalized by $P_{i}$ which is the amplitude of the pressure fluctuation at the microphone located at $\theta=90^{\circ}$ for the simulations without the turbulent layer. The scattered field is quite complex (compared to the scattered fields observed in [10] for a single vortex), because of interferences between the scattered fields radiated by each turbulent structure/volume. The levels of scattered pressure appear to be roughly an order of magnitude lower than the total pressure fluctuations. On this snapshot of the scattered field, it seems that some bundles of large turbulent structures are scattering quite efficiently (for example, the turbulent area between $1 \delta \leq x \leq 3$ ). This spatial distribution of scattered pressure is evolving with time since the turbulence is convected, but the scattered levels in the upstream direction appear to be more important. This can be explained in part because the incoming radiation from 
the monopole is more intense upstream due to the convective amplification, but also because the wavelength of the incident field is smaller upstream and thus, the scattering is expected to be more intense (as observed in [10] for the scattering by a single vortex). In Fig. 4, we can also notice that the backscattering (towards the $-y$-direction) appears to be weak by comparison with the forward scattering. The study realized in [10] for a single vortex shows that the backscattering is expected to be important relative to the forward scattering for very low incident frequencies, but rapidly disappears as the frequency of the source increases.

Fig. 5 is similar to Fig. 4 but for a high frequency source $\left(f_{0}=8 c_{0} / \delta\right)$. The scattering is more intense and strongly affects the total pressure field. The total and scattered fields have the same order of magnitude and, as expected, no backscattering is observable for this frequency.

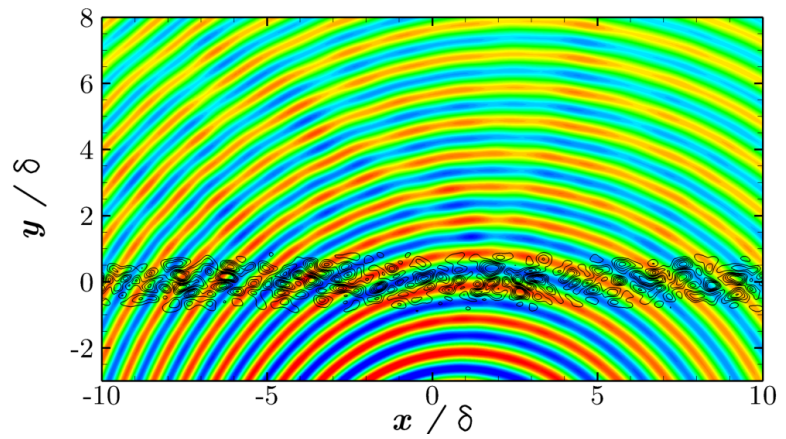

(a)

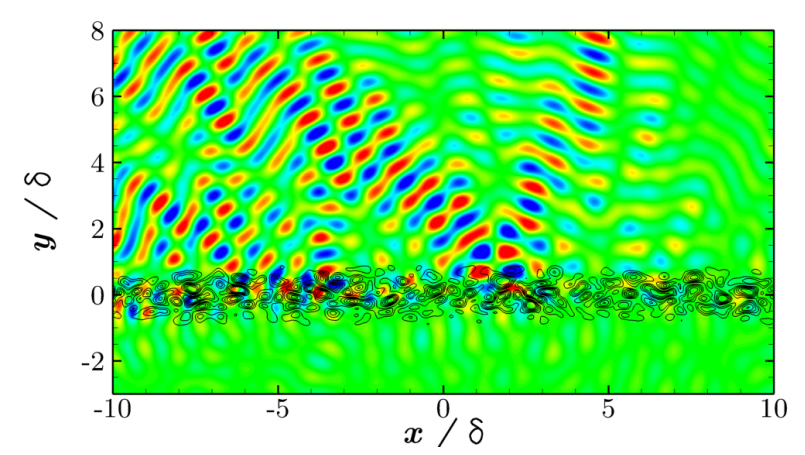

(b)

Figure 4: Snapshots of $(a)$ the total pressure fluctuation $p^{\prime} / P_{i}$ (levels between \pm 1.5$)$ and $(b)$ the scattered pressure fluctuation $p_{s}^{\prime} / P_{i}$ (levels between \pm 0.15 ) for the source frequency $f_{0}=c_{0} / \delta$. The lines are the $u_{t} / c_{0}$ turbulent velocity component (levels between \pm 0.05 ).

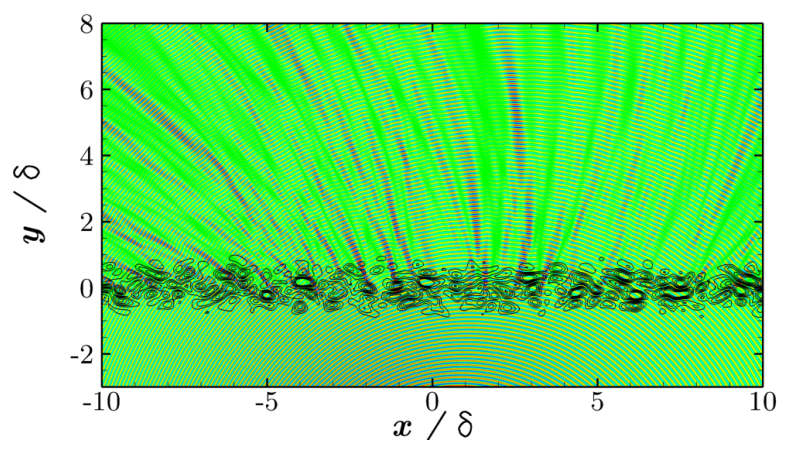

(a)

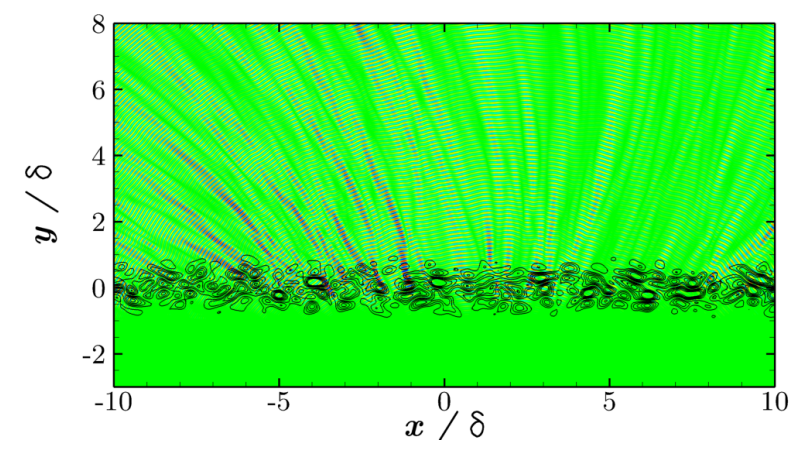

(b)

Figure 5: Snapshots of $(a)$ the total pressure fluctuation $p^{\prime} / P_{i}$ (levels between \pm 2 ) and $(b)$ the scattered pressure fluctuation $p_{s}^{\prime} / P_{i}$ (levels between \pm 2 ) for the source frequency $f_{0}=8 c_{0} / \delta$. The lines are the $u_{t} / c_{0}$ turbulent velocity component (levels between \pm 0.05 ).

Fig. 6 presents time signals of the total pressure fluctuation for the two cases presented in Figs. 4 and 5 at the microphone position $\theta=90^{\circ}$. The signals are plotted over a time duration that corresponds to the length of the segments used in the periodograms for the calculation of the SPL presented in the rest of the paper. These signals appear to have been modulated, at least in amplitude, by the scattering phenomenon and the extent of this modulation seems relatively small for the low frequency case but very strong at high frequency, similarly to the observations made on the previous snapshots. As the Doppler effect is thought to be one of the main mechanism of the spectral broadening, a frequency modulation of the scattered field can also be expected, but this phenomenon is less trivial to observe.

The pressure time signals recorded on the circular array are used to compute the SPL. The transient period required for the monopole radiation to propagate across the domain at the beginning of the calculation is removed from the signals before performing a periodogram. All the SPL presented throughout the paper are calculated using the same segment length in the periodogram and thus, have the same frequency 


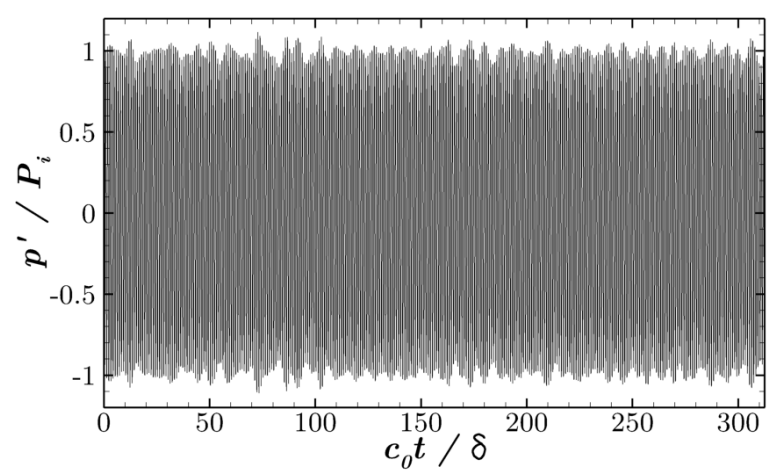

(a)

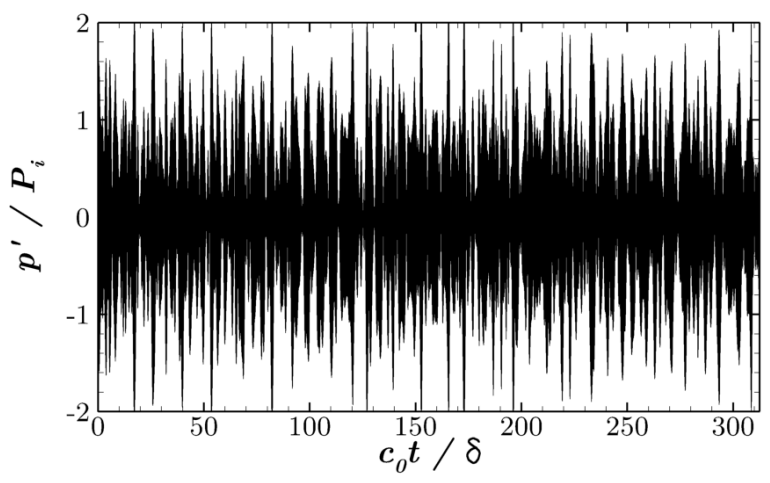

(b)

Figure 6: Pressure fluctuation signals over the duration of a segment of the periodogram at the microphone position $\theta=90^{\circ}$ for the source frequencies $(a) f_{0}=c_{0} / \delta$ and $(b) f_{0}=8 c_{0} / \delta$.

resolution. A Hann window is used and the segment overlapping in the periodogram is set to 50\%. For the high frequency simulations, since the time-step is smaller to preserve the CFL condition, the number of segments averaged for the calculation of the SPL are lower. As a consequence, the spectra appear to be more oscillating for the high frequency cases.

The SPL calculated at $\theta=90^{\circ}$ for the five different frequency considered are presented in Fig. 7. The levels are normalized by the levels of the peaks at $f_{0}$ obtained from the simulations carried out without the turbulent layer. All the spectra display sidebands around the peak at the source frequency. The levels of the sidebands are increasing with the frequency, which is in agreement with observations previously made in previous experimental and numerical work. ${ }^{7,8,17,24,30}$ The levels of the peaks are also affected by the scattering. At low frequency, the sidebands levels are very low (the scattering is weak) and the peak is almost unchanged. As the frequency increases, the reductions in the peak level are also increasing and reach more than $3 \mathrm{~dB}$ for the highest frequency considered. This evolution of the peak level with the source frequency has also been observed experimentally in the literature.

In Fig. 7(a), it appears that the sidebands are getting wider when the source frequency increases. If the spectra are now plotted as a function of the frequency reduced by the source frequency $\left(f-f_{0}\right) / f_{0}$ as in Fig. 7(b), we can observe that, relative to the source frequency, the sidebands are getting narrower as the source frequency increases. In the context of a Doppler shift based explanation, this means that the scattered field received by the microphone is coming from angles around the source-microphone line that are narrower as the source frequency is increased. This implies that the scattering angular range of the turbulent structures is becoming narrower around the incident wave direction when the frequency increases. This conclusion is in complete agreement with the results of [10] obtained for the scattering of acoustic waves by a single vortex.

Some differences can be observed with previous experimental results in terms of the evolution of the positions of the sidebands maxima with the source frequency. In most experimental results, the positions of the sidebands maxima seems to remain constant with the source frequency. ${ }^{7,24,30}$ In Fig. 7 (a) this seems to hold for the two or three highest source frequencies, but the sidebands are moving significantly for the lowest frequencies. It is suggested here that this is not observed experimentally, even in the configurations where the source wavelength to turbulent layer thickness ratio $\left(\lambda_{0} / \delta\right)$ is $\geq 1$, because of the difference in the spectral content of the turbulence in the layer between these experiments and the present study.

The turbulent macro-scale parameter $l$ that is defined in previous work ${ }^{7,26,30}$ is calculated here for the high frequency sources, since the experimental trends observed for the positions of the sidebands holds for these cases. This macro-scale is defined:

$$
l=\frac{U_{0}}{\Delta f}
$$

where $\Delta f$ is the position of the maximum of a sideband relative to the peak. Note that the definition of Candel et al. ${ }^{7}$ or Sijtsma et al. ${ }^{30}$ is $l=0.5 U_{0} / \Delta f$ because the convection velocity of the turbulent structures 
in the shear layer is estimated as $0.5 U_{0}$. In the present study, the mean flow is uniform and the convection velocity is $U_{0}$. In Sijtsma et al., ${ }^{30}$ where results from different wind tunnels are compared, it is argued that the ratio $l / \delta_{C A}$ lies between 3.2 and 4.4, where $\delta_{C A}$ is the shear layer thickness defined in [30] Eq.(18).

The definition of $\delta$ from the turbulent kinetic energy distribution expressed in Sec. III:

$$
k_{t}\left( \pm \frac{\delta}{2}\right)=\frac{k_{t, \max }}{4}
$$

seems to fit roughly the values of $\delta_{C A}$ for several of the wind tunnels reported by Sijtsma et al. ${ }^{30}$ thus $\delta_{C A} \approx \delta$. In Fig. 7, the maxima of the sidebands lie at $\Delta f \delta / c_{0} \approx 0.2$, thus the ratio $l / \delta=\mathrm{M} c_{0} /(\Delta f \delta) \approx 0.88$ which is largely below the 3.2-4.4 range reported in previous experiments. That means that the macro-scale of the turbulence in the present study is significantly smaller than in these experiments, which can explain some differences observed for low frequency sources where the ratio between the source acoustic wavelength and the turbulence scales is larger than the experimental ones.

A final remark can be made on the shape of the spectra in Fig. 7(a) when compared to the results of a previous study by the authors on the scattering by a single vortex convected in a uniform mean flow, presented in Fig. 8. It can be seen that the shapes of the spectra calculated from the present simulations for the low frequency sources $\left(f_{0}=c_{0} / 2 \delta\right.$ and $\left.f_{0}=c_{0} / \delta\right)$ are very similar to the one observed for a single vortex for the low frequency sources. But as the frequency increases, the SPL for the scattering by a turbulent layer seem to transition and become very similar to the one observed in previous experimental studies. ${ }^{7,24,30}$ It seems that for a low frequency source, the acoustic waves are scattered by the turbulent layer as if there was a single scale within the width of the layer, whereas at high frequency the acoustic waves are more sensitive to the fine structure of the turbulence, leading to a different scattering spectrum (possibly including multiple scattering). Further work is required on this transition to fully understand its underlying mechanisms.

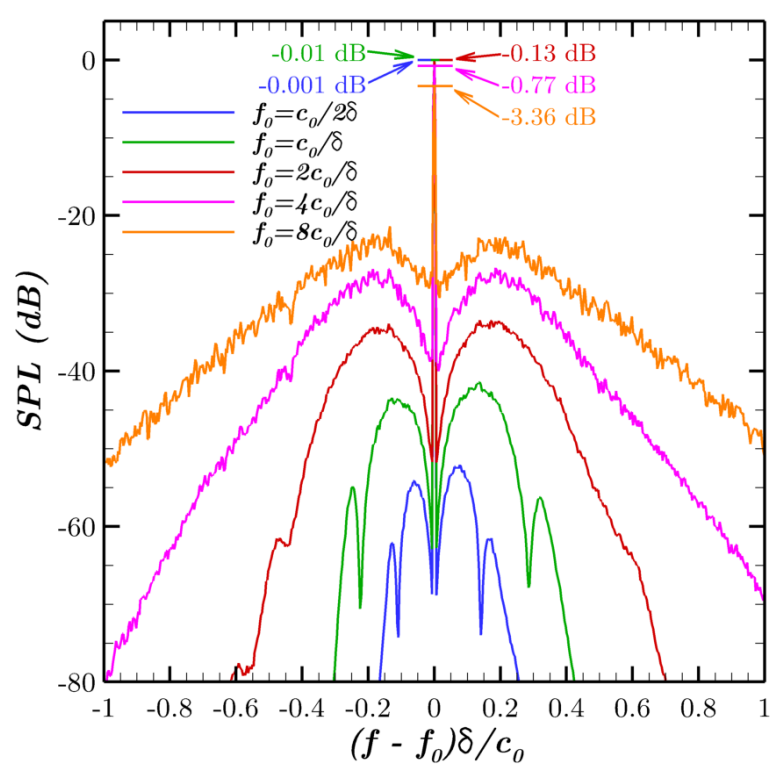

(a)

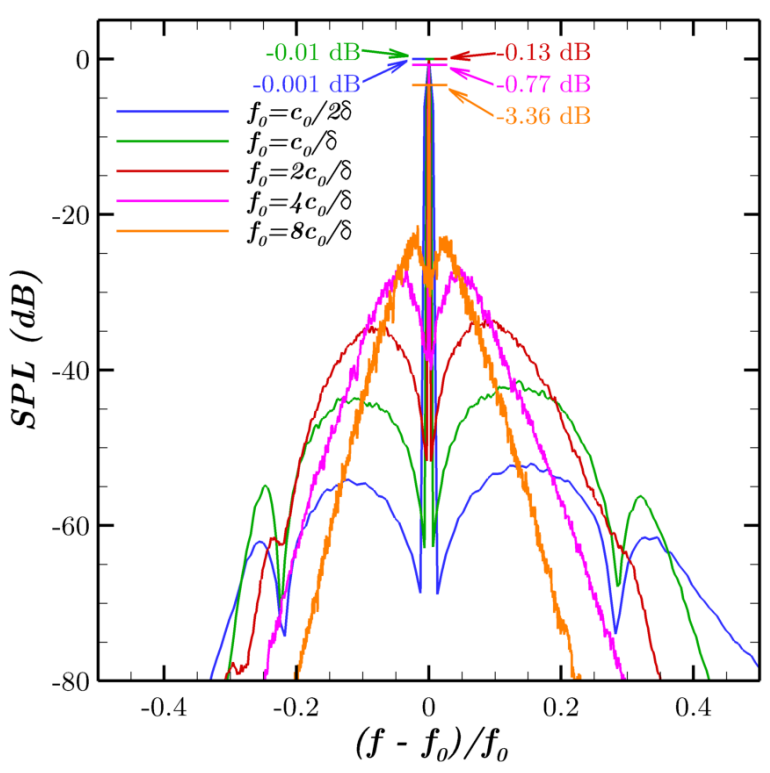

(b)

Figure 7: Comparison of the SPL at the microphone position $\theta=90^{\circ}$ for different source frequencies $(a)$ plotted versus the frequency and $(b)$ plotted versus the frequency reduced by the source frequency $f_{0}$.

Fig. 9 presents the SPL at 3 different microphone positions on the circular array for a low frequency and a high frequency source. Note that, in most of the existing experimental results, the scattered levels are increasing when the microphone is moved downstream. This can be related to the fact that the configurations studied experimentally are spreading shear layers and thus, the wave propagating downstream have to cross a larger shear layer (possibly containing large eddies) and are more scattered. This is not the case in the present configuration, since a constant width turbulent layer is considered. The maximum of the sidebands 


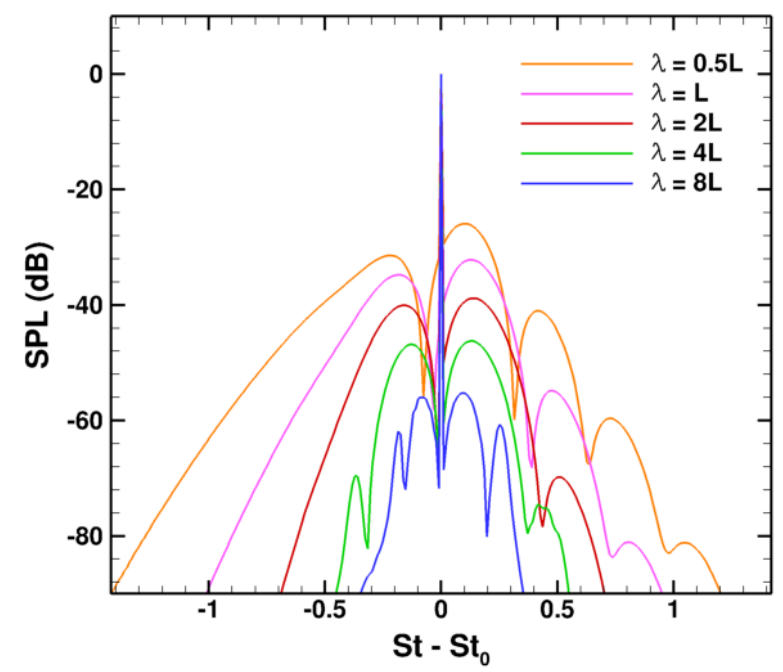

Figure 8: SPL obtained for the scattering by a single inviscid vortex of core size $L$ convected by a uniform mean flow at $\mathrm{M}=0.176$ for different source frequencies (or wavelength in the legends). Results from Clair and Gabard. ${ }^{10}$

is even higher for the microphone located upstream and, on the high frequency case, the peak reduction is also larger upstream, probably because of the convection effects on the incident radiation.

For the low frequency source, the secondary lobes are evolving significantly with the position of the microphone. These evolutions can be related in terms of the Doppler effect. In the study of the scattering by a convected vortex, ${ }^{10}$ it has been shown that the scattering emitted by turbulent structures located upstream of the source-microphone emission line will contribute to the high frequency sideband, whereas the structures located downstream will contribute to the low frequency sideband. This is explained by the fact that depending on the position of the structure, it is moving towards or away from the microphone and thus, the Doppler shift is positive or negative. Additionally, the further a structure is from the source-microphone emission line, the larger the Doppler shift will be. For the downstream microphone $\left(\theta=50^{\circ}\right)$, there is a larger volume of turbulence located upstream than downstream and this explains why there is more content with a large positive frequency shift (the secondary lobe between $\left.0.24 \leq\left(f-f_{0}\right) \delta / c_{0} \leq 0.45\right)$ but no secondary lobe on the low frequency side. A similar reasoning can be made for the microphone located upstream, showing a low frequency secondary lobe, but none at high frequency.

Note that these observations are relevant for a low frequency source, when the turbulent structures are expected to have a wide scattering directivity. For a high frequency source, the turbulent structures are scattering over a smaller angular range around the emission line thus the spectra do not display such differences in the frequency content. In Fig. 9(b), it even appears that the high frequencies are decaying faster downstream and the low frequencies upstream. 


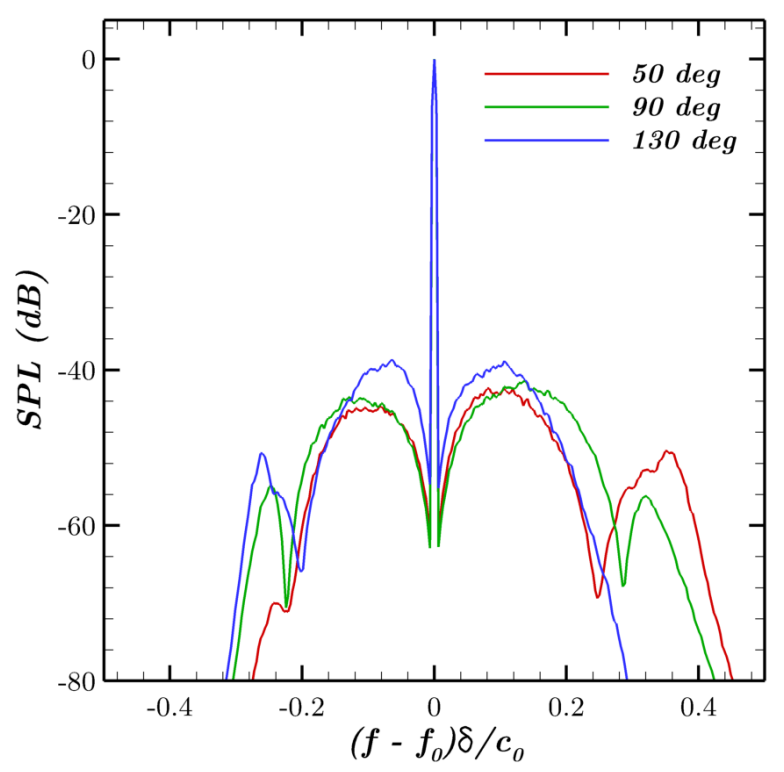

(a)

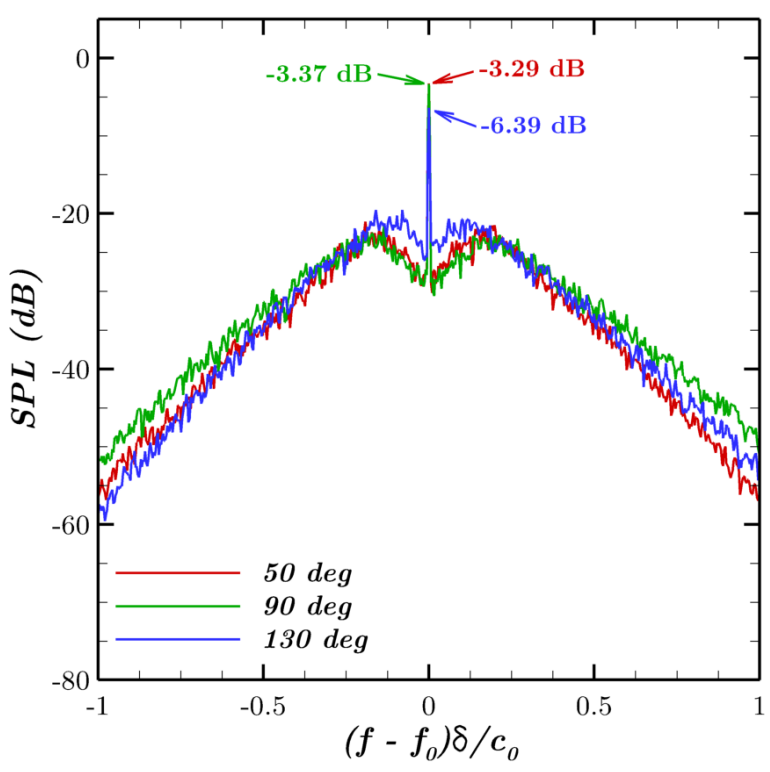

(b)

Figure 9: Comparison of the SPL at different microphone positions for the cases $(a) f_{0}=c_{0} / \delta$ and $(b)$ $f_{0}=8 c_{0} / \delta$.

\section{IV.B. Effects of the convection velocity}

Simulations have been run for three different Mach numbers for two source frequencies $\left(f_{0}=c_{0} / \delta\right.$ and $\left.f_{0}=c_{0} / \delta\right)$. The SPL at $\theta=90^{\circ}$ for the low frequency source are presented in Fig. 10. Increasing the convection velocity seems to lead to a widening of the sidebands in Fig. 10(a) and an increase of the scattered levels. These observation are in agreement both with previous experimental work, and with the study of the scattering by a single vortex. The increase of the levels is explained by the increase of the turbulent velocities in the layer since the turbulent intensity is kept at $15 \%$ for all the mean velocities considered. The same spectra are plotted as a function of the Strouhal number defined as $S t=f \delta / U_{0}$ in Fig. 10(b) and it appears that the position of the sidebands is converging quite well for the three velocities considered, at least for the main lobe. This suggests that the position of the sidebands is evolving linearly with the convection velocity, which is consistent with an explanation of the spectral broadening based on a Doppler shift. In Fig. 10(b), the differences remaining on the spectra such as the narrowing of the sidebands and the change in the positions of the secondary lobes when the Mach number is increased might be due to a combination of the effects of the convection on the incident radiation and of the evolution of the spatial distribution of the scattered field when the turbulent velocities are increased.

Fig. 11 presents the results for the high frequency source. From $\mathrm{M}=0.088$ to $\mathrm{M}=0.176$ the scattered levels are increased but in a lesser proportion than for the low frequency source presented before. The position of the sidebands is still evolving linearly with the convection velocity. The decay of the spectra away from the sidebands appears to be slower at $\mathrm{M}=0.176$ than at $\mathrm{M}=0.088$. For the highest Mach number, the shape of the spectrum has completely changed. Instead of sidebands on each side of the peak at the source frequency, the spectrum consists of one broad hump surrounding the peak and decaying slowly. By looking at the peak reduction $(\approx-18 \mathrm{~dB})$, it appears that a case of strong scattering has been reached.

This case $\left(f_{0}=c_{0} / \delta\right.$ and $\left.\mathrm{M}=0.352\right)$ is further detailed by looking at the SPL for different microphones, as plotted in Fig. 12. The shape of the spectra remains relatively similar for the three microphones considered but, for the microphone located upstream, the peak seems to have completely disappeared within the broadband hump. Away from the peak, the scattered levels seem to be the highest for $\theta=90^{\circ}$ both at high and low frequencies. 


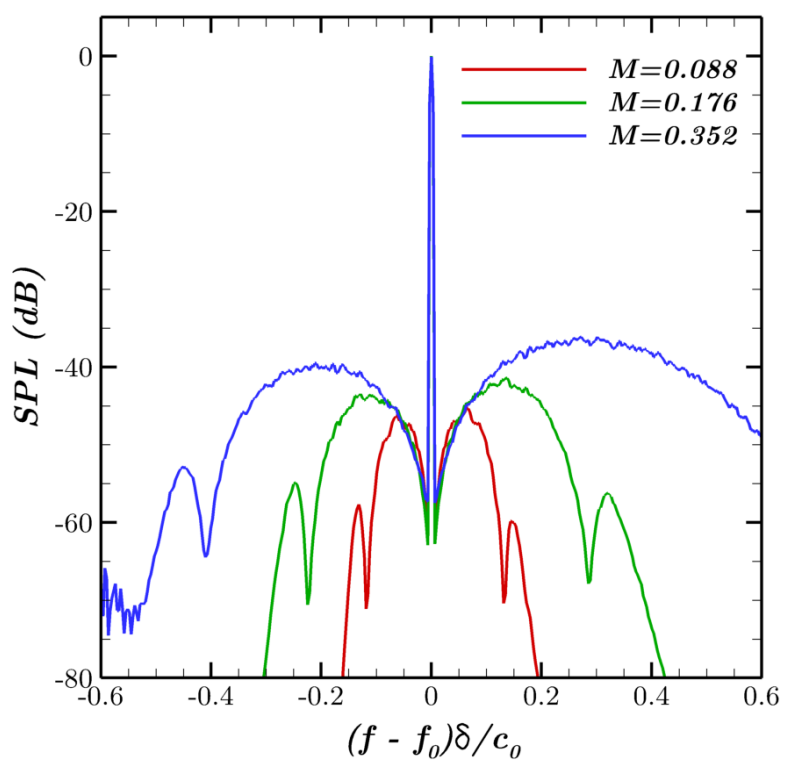

(a)

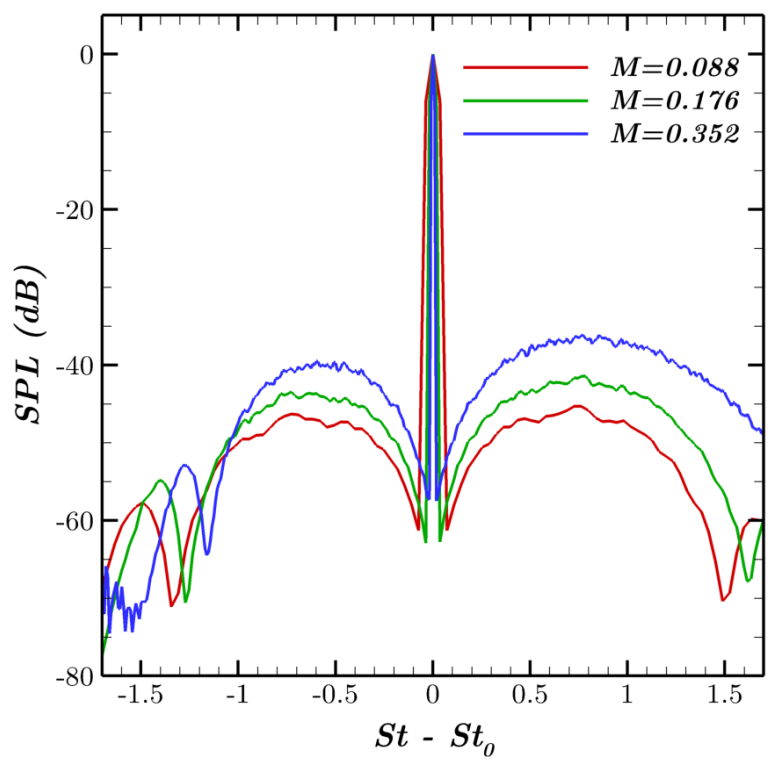

(b)

Figure 10: Comparison of the SPL at different convection Mach numbers for a source frequency $f_{0}=c_{0} / \delta$ (a) plotted versus the frequency and (b) plotted versus the Strouhal number $S t=f \delta / U_{0}$.

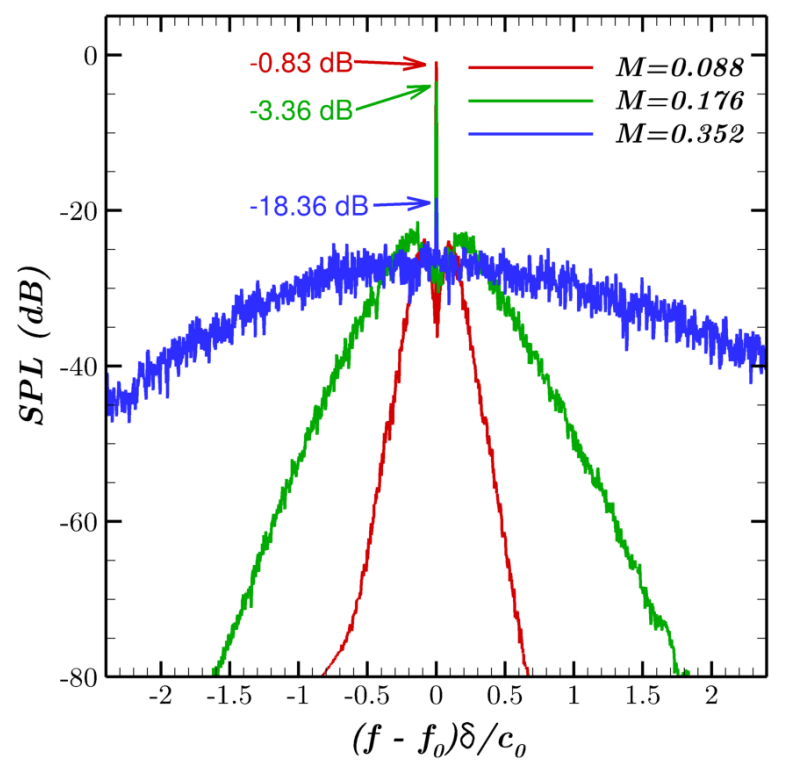

(a)

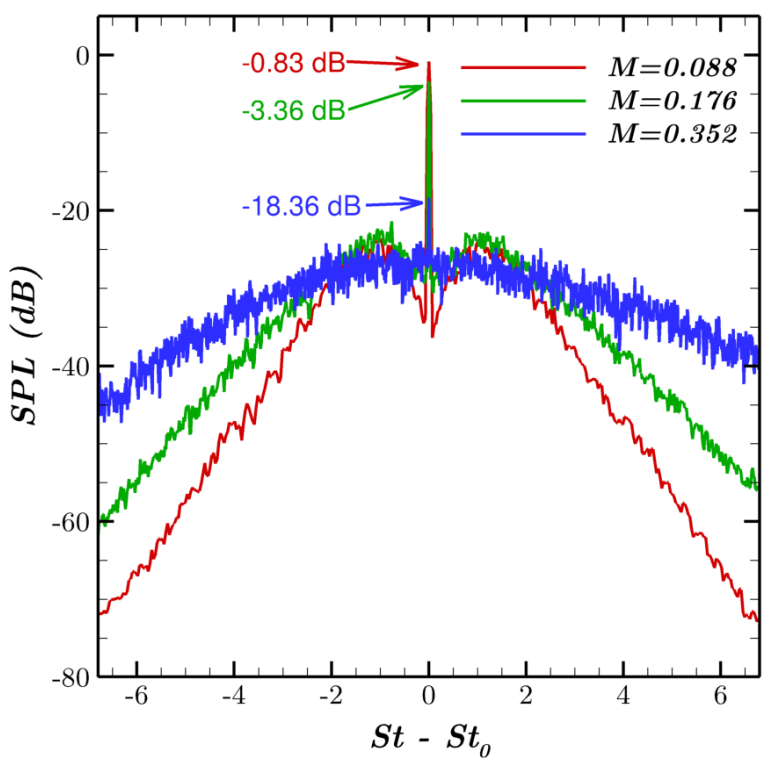

(b)

Figure 11: Comparison of the SPL at different convection Mach numbers for a source frequency $f_{0}=8 c_{0} / \delta$ (a) plotted versus the frequency and (b) plotted versus the Strouhal number $S t=f \delta / U_{0}$. 


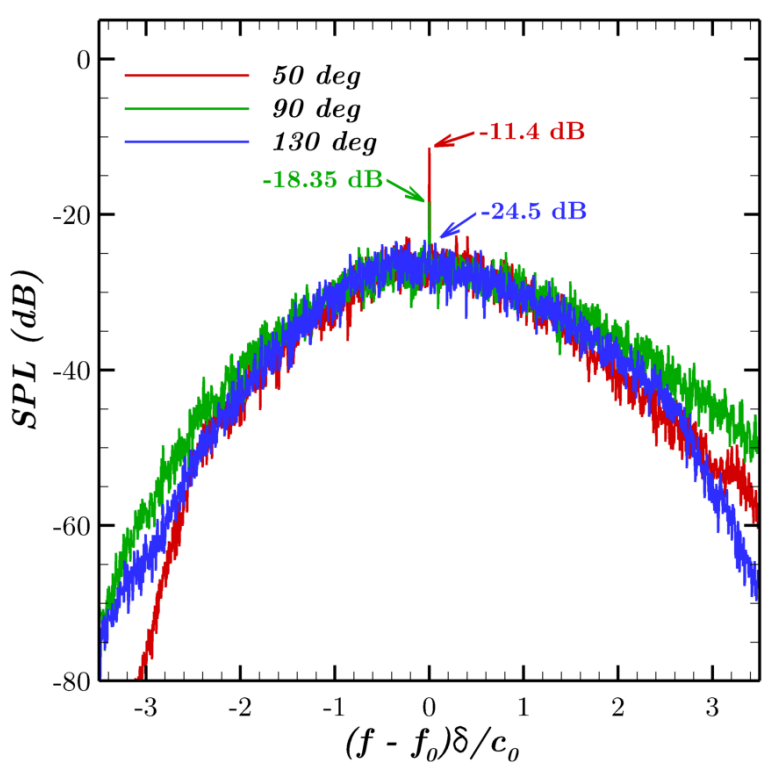

Figure 12: Comparison of the SPL at different microphone positions for the case $f_{0}=8 c_{0} / \delta$ at $\mathrm{M}=0.352$.

\section{Conclusion}

The scattering of a harmonic monopole by a turbulent layer with constant width and convected by a uniform flow has been studied numerically. The computational method used is based on the linearized Euler equations in the time domain and a stochastic method to synthesize turbulent fluctuations by filtering a white noise. The computations presented in this paper are focused on the effects of the source frequency and the mean flow velocity on the scattering. The scattered pressure fields show a lot of interferences due to the simultaneous scattering of all the structures comprised in the turbulent layer. The total pressure field appear to be strongly affected by the scattering when a high frequency source is considered.

The acoustic spectra deduced from these simulations display sidebands surrounding the incident frequency peak. For a low frequency source, these spectra are very similar to the one observed in previous work on the scattering of a harmonic source by a single convected vortex. For a high frequency source, the results show strong similarities with the sidebands observed in previous studies of the spectral broadening by a jet shear layer. The transition between these two regimes are required to determine if phenomenon like multiple scattering are at play. The differences in the evolution of the sideband position between the present results for low frequency sources and experimental results with similar acoustic wavelength to turbulent layer thickness ratio have been attributed to differences in the frequency contents of the turbulence.

The trends deduced from these computations can be related to previous results and correlate quite well with the hypothesis that the spectral broadening is linked to a Doppler shift due to the movement of the turbulent eddies acting as sources of the scattered field. This is observed here with the linear widening of the sidebands with the convection velocity, and also with the appearance of high or low frequency content on the spectra depending on the position of the microphone (for a low frequency source). Increasing the frequency of the source leads to a widening of the sidebands when the SPL are plotted versus the frequency, but they actually become narrower relative to the frequency of the source. This is again in agreement with a Doppler effect hypothesis, since previous work showed that at higher source frequency, the scattering by an eddy is occurring over a narrower angular range around the incident wave propagation direction, leading to smaller relative frequency shifts.

Additionally, an increase in the source frequency leads to higher scattered levels combined with a stronger reduction of the levels of the peak at the source frequency. For the highest source frequency and convection velocity considered, strong scattering is observed where the peak undergo large reductions in its levels. The peak even completely disappears for microphones located upstream. For this case, the shape of the scattered spectra becomes a single broadband hump centered on the peak frequency. 
Future work with the same simplified configuration includes a parametric study of the effects of the turbulence parameters. These have received very few attention so far, since they are difficult to control experimentally. The advantage of using a stochastic method is that some parameters can be easily changed, such as the turbulent intensity, the turbulent layer width, the integral length scale and the correlation time (only frozen turbulence has been considered here). It also possible to consider different turbulence spectra (or spatial correlations). Further work in the analysis of these results is also ongoing to gain insight on the physical mechanisms involved in the spectral broadening. This includes post-treatment of the numerical results using correlations, beamforming techniques and higher order statistical methods.

\section{Acknowledgements}

This work was partly supported by Rolls-Royce plc through the University Technology Center in Gas Turbine Noise at the University of Southampton and funded by EPSRC Grant EP/K017551/1.

The authors would like to acknowledge the members of the AS/TA department of DLR-Braunschweig for providing the PIANO solver and the technical support associated.

The authors also acknowledge the use of the IRIDIS High Performance Computing Facility, and associated support services at the University of Southampton, in the completion of this work.

\section{References}

\footnotetext{
${ }^{1}$ Bennaceur, I., Mincu, D., Mary, I., Terracol, M., Larchevêque, L. \& Dupont, P., "Numerical simulation of spectral broadening of an acoustic wave by a spatially growing turbulent mixing layer", 21st AIAA/CEAS Aeroacoustics Conference, $\mathrm{n}^{\circ}$ AIAA-2015-2841, 2015.

${ }^{2}$ Brown, E., "Turbulent spectral broadening of backscattered acoustic pulses", Journal of the Acoustical Society of America, 56(5), 1974, pp. 1398-1406.

${ }^{3}$ Brown, E. \& Clifford, S., "Spectral broadening of an acoustic pulse propagating through turbulence", Journal of the Acoustical Society of America, 54(1), 1973, pp. 36-39.

${ }^{4}$ Brown, E. \& Clifford, S., "On the attenuation of sound by turbulence", Journal of the Acoustical Society of America, 60(4), 1976, pp. 788-794.

${ }^{5}$ Campos, L., "The spectral broadening of sound by turbulent shear layers. Part 1. The transmission of sound through turbulent shear layers", Journal of Fluid Mechanics, 89(4), 1978, pp. 723-749.

${ }^{6}$ Campos, L., "The spectral broadening of sound by turbulent shear layers. Part 2. The spectral broadening of sound and aircraft noise", Journal of Fluid Mechanics, 89(4), 1978, pp. 751-783.

${ }^{7}$ Candel, S., Guedel, A. \& Julienne, A., "Refraction and scattering of sound in an open wind tunnel flow", 6th International Congress on Instrumentation in Aerospace Simulation Facilities, 1975, pp. 288-300.

${ }^{8}$ Candel, S., Guedel, A. \& Julienne, A., "Radiation, refraction and scattering of acoustic waves in a free shear flow", 3rd AIAA Aero-Acoustics Conference, 1976.

${ }^{9}$ Cheinet, S., Ehrhardt, L., Juvé, D. \& Blanc-Benon, P., "Unified modeling of turbulence effects on sound propagation", Journal of the Acoustical Society of America, 132(4), 2012, pp. 2198-2209.

${ }^{10}$ Clair, V., Gabard, G., "Numerical assessment of the scattering of acoustic waves by turbulent structures", 21st AIAA/CEAS Aeroacoustics Conference, n AIAA-2015-2680, 2015.

${ }^{11}$ Dallois, L., Blanc-Benon, P. \& Juvé, D., "A wide-angle parabolic equation for acoustic waves in inhomogeneous moving media: applications to atmospheric sound propagation", Journal of Computational Acoustics, 9(2), 2001, pp. 477-494.

${ }^{12}$ Dieste, M., Gabard, G., "Random particle methods applied to broadband fan interaction noise", Journal of Computational Physics, 231, 2012, pp. 8133-8151.

${ }^{13}$ Ehrhardt, L., Cheinet, S., Juvé, D. \& Blanc-Benon, P., "Evaluating a linearized Euler equations model for strong turbulence effects on sound propagation", Journal of the Acoustical Society of America, 133(4), 2013, pp. $1922-1933$.

${ }^{14}$ Ewert, R., "Broadband slat noise prediction based on CAA and stochastic sound sources from a fast Random ParticleMesh (RPM) method", Computers and Fluids, 37, 2008, pp. 369-387.

${ }^{15}$ Ewert, R., Dierke, J., Siebert, J., Neifeld, A., Appel, C., Siefert, M. \& Kornow, O., "CAA broadband noise prediction for aeroacoustic design", Journal of Sound and Vibration, 330, 2011, pp. 4139-4160.

${ }^{16}$ Ewert, R., Kornow, O., Delfs, J., Yin, J., Röber, T. \& Rose, M., "A CAA based approach to tone haystacking", 15th AIAA/CEAS Aeroacoustics Conference, n AIAA-2009-3217, 2009

${ }^{17}$ Ewert, R., Kornow, Tester, B., Powles, C., Delfs, J. \& Rose, M., "Spectral broadening of jet engine turbine tones", $14 t h$ AIAA/CEAS Aeroacoustics Conference, n AIAA-2008-2940, 2008.

${ }^{18}$ Ewert, R. \& Schröder, W. "On the simulation of trailing edge noise with a hybrid LES/APE method", Journal of Sound and Vibration, 270, 2004, pp. 509-524.

${ }^{19}$ Ford, R. \& Llewellyn Smith, S., "Scattering of acoustic waves by a vortex", Journal of Fluid Mechanics, 386, 1999, pp. 305-328.

${ }^{20}$ Goedecke, G., Wood, R., Auvermann, H., Ostashev, V., Havelock, D. \& Ting, C., "Spectral broadening of sound scattered by advecting atmospheric turbulence", Journal of the Acoustical Society of America, 109(5), 2001, pp. 1923-1934.
} 
${ }^{21}$ Guédel, A., "Scattering of an acoustic field by a free shear layer", Journal of Sound and Vibration, 100(2), 1985, pp. 285-304.

${ }^{22}$ Howe, M., "Multiple scattering of sound by turbulence and other inhomogeneities", Journal of Sound and Vibration, 27(4), 1973, pp. 455-476.

${ }^{23}$ Kraichnan, R., "The scattering of sound in a turbulent medium", Journal of the Acoutical Society of America, 25(6), 1953, pp. 1096-1104.

${ }^{24}$ Kröber, S., Hellmold, M. \& Koop, L., "Experimental Investigation of Spectral Broadening of Sound Waves by Wind Tunnel Shear Layers", 19th AIAA/CEAS Aeroacoustics Conference, n AIAA-2013-2255, 2013.

${ }^{25}$ Lighthill, M., "On the energy scattered from the interaction of turbulence with sound or shock waves", Mathematical Proceedings of the Cambridge Philosophical Society, 49, 1953, pp. 531-551.

${ }^{26}$ McAlpine, A., Powles, C. \& Tester, B., "A weak-scattering model for turbine-tone haystacking", Journal of Sound and Vibration, 332, 2013, pp. 3806-3831.

${ }^{27}$ Ostashev, V., Salomons, E., Clifford, S., Lataitis, R., Wilson, D., Blanc-Benon, P. \& Juvé, D., "Sound propagation in a turbulent atmosphere near the ground: A parabolic equation approach", Journal of the Acoustical Society of America, 109(5), 2001, pp. 1894-1908.

${ }^{28}$ Powles, C., Tester, B. \& McAlpine, A., "A weak-scattering model for turbine-tone haystacking outside the cone of silence", International Journal of Aeroacoustics, 10(1), 2011, pp. 17-50.

${ }^{29}$ Salomons, E., Ostashev, V., Clifford, S. \& Lataitis, R., "Sound propagation in a turbulent atmosphere near the ground: An approach based on the spectral representation of refractive-index fluctuations", Journal of the Acoustical Society of America, 109(5), 2001, pp. 1881-1893.

${ }^{30}$ Sijtsma, P., Oerlemans, S., Tibbe, T., Berkefeld, T. \& Spehr, C., "Spectral broadening by shear layers of open jet wind tunnels", 20th AIAA/CEAS Aeroacoustics Conference, n AIAA-2014-3178, 2014.

${ }^{31}$ Tam, C. \& Webb, J., "Dispersion-Relation-Preserving finite difference schemes for computational acoustics", Journal of Computational Physics, 107(2), 1993, pp. 262-281.

${ }^{32}$ Wilson, D., Ostashev, V., Goedecke, G. \& Auvermann, H., "Quasi-wavelet calculations of sound scattering behind barriers", Applied Acoustics, 65, 2004, pp. 605-627. 